\title{
Shape Gradients for Histogram Segmentation using Active Contours
}

\author{
Stéphanie Jehan-Besson Michel Barlaud \\ Laboratoire I3S, CNRS-UNSA \\ 2000, route des Lucioles \\ 06903 Sophia Antipolis, France \\ \{jehan,barlaud\}@i3s.unice.fr *
}

\author{
Gilles Aubert \\ Laboratoire J.A. Dieudonné, CNRS-UNSA \\ Parc Valrose \\ 06108 Nice Cedex 2, France \\ gaubert@math.unice.fr
}

\author{
Olivier Faugeras \\ INRIA Sophia Antipolis, 2004 route des Lucioles, BP 93 \\ 06902, Sophia-Antipolis Cedex, France. \\ faugeras@sophia.inria.fr
}

\begin{abstract}
We consider the problem of image segmentation using active contours through the minimization of an energy criterion involving both region and boundary functionals. These functionals are derived through a shape derivative approach instead of classical calculus of variation. The equations can be elegantly derived without converting the region integrals into boundary integrals. From the derivative, we deduce the evolution equation of an active contour that makes it evolve towards a minimum of the criterion. We focus more particularly on statistical features globally attached to the region and especially to the probability density functions of image features such as the color histogram of a region. A theoretical framework is set for the minimization of the distance between two histograms for matching or tracking purposes. An application of this framework to the segmentation of color histograms in video sequences is then proposed. We briefly describe our numerical scheme and show some experimental results.
\end{abstract}

\section{Introduction}

Active contours are powerful tools for image and video segmentation or tracking. They can be formulated in the framework of variational methods. The basic principle is to construct a PDE (Partial Differential Equation) from an energy criterion, including usually both region and boundary functionals, by computing some sort of Euler-Lagrange equations; this PDE changes the shape of the current curve

* This work was supported by France Telecom R\&D grants nb 011BC22 and $021 \mathrm{~B} 406$ according to some velocity field which can be thought of as a descent direction of the energy criterion. Given a closed curve enclosing an initial region, one then computes the solution of this PDE for this initial condition. The corresponding family of curves decreases the energy criterion and converges toward a (local) minimum of the criterion hopefully corresponding to the objects to be segmented.

Originally, snakes [1], balloons [2] or geodesic active contours [3] are driven towards the edges of an image through the minimization of a boundary integral of features depending on edges. Active contours driven by the minimization of region functionals in addition to boundary functionals have appeared later. Introduced by [4] and [5], they have been further developed in $[6,7,8,9,10,11,12]$. In effect, the use of active contours for the optimization of a criterion including both region and boundary functionals appears to be powerful.

However, the PDE computation is not trivial when the energy criterion involves region functionals. This is mostly due to the fact that the set of image regions does not have a structure of vector space, preventing us to use in a straightforward fashion gradient descent methods. To circumvent this problem, many methods have been proposed in the literature. Some authors do not compute the theoretical expression of the velocity field (basically the gradient of the energy criterion) but choose a deformation of the curve that will make the criterion decrease $[7,8]$ (they compute a direction of descent). Other authors $[6,10]$ compute the theoretical expression of the velocity vector from the EulerLagrange equations. First, region integrals are transformed into boundary integrals using the Green-Riemann theorem. Second, the corresponding Euler-Lagrange equations are derived, and used to define a dynamic scheme to evolve the initial region. Another alternative is to compute the gra- 
dient directly from the region formulation without reducing it to boundary integrals. In [13], a level set formulation is introduced directly in the criterion and in [11], the authors propose to compute the derivative of the criterion while taking into account the discontinuities across the contour. In $[14,15,16]$ the computation of the evolution equation is achieved through shape derivation principles.

This computation becomes more involved when global information about regions is present in the energy criterion, the so-called region-dependent case. It happens when statistical features of a region such as, for example, the mean or the variance of the intensity, are involved in the minimization. Some region-dependent features have been successfully used in $[13,11,12,17]$ for segmentation and classification. In $[14,15,16]$ we propose a general framework based on shape derivation tools for the computation of the related evolution equation.

In this article, we first recall shape derivation principles to construct the evolution equation from the energy criterion including both region and boundary functionals. We then turn our attention to statistical region-based functionals based on probability density functions of image features. In [17], such features have been used to maximize the mutual information between a binary region label and the intensity value, for nonparametric segmentation. We propose here an energy criterion that minimizes the distance between the probability density functions of the current region and the reference one, and we consider regions tracking or matching applications. The shape derivation tools allow us to easily derive the velocity field that defines the evolution of the region boundary. The global evolution equation is then given for first and second order histograms. This theoretical framework can be applied to the problem of region segmentation using a given reference color histogram in a sequence of images. The region defined by the active contour evolves so that the distance between the current region histogram and the reference one decreases, allowing us to segment the region of interest. Experimental results are provided including the visualization of the evolution of region histograms during the propagation of the curve.

Region and boundary functionals are presented in section 2 while shape derivation tools are presented in section 3. The theoretical framework for the minimization of region functionals involving the distance between probability density functions is set in section 4 . The application to region segmentation on videos using color histograms is detailed in section 5 .

\section{Problem Statement}

In many image processing problems, the issue is to find a set of image regions that minimize a given error criterion. This criterion is often a combination of region and boundary functionals. The basic idea is to derive a Partial Differential Equation (PDE) that will drive the boundary of an initial region towards a local minimum of the error criterion. The key point is to compute the velocity vector at each point of the boundary at each time instant.

To fix ideas, in the two-dimensional case, the evolving boundary, or active contour, is modeled by a parametric curve $\Gamma(s, \tau)=\left(x_{1}(s, \tau), x_{2}(s, \tau)\right)$, where $s$ may be its arc-length and $\tau$ is an evolution parameter, the time. The active contour is then driven by the following PDE:

$$
\Gamma_{\tau} \stackrel{\text { def }}{=} \frac{\partial \Gamma(s, \tau)}{\partial \tau}=\mathbf{v} \quad \text { with } \Gamma(\tau=0)=\Gamma_{0},
$$

where $\Gamma_{0}$ is an initial curve defined by the user and $\mathbf{v}$ the velocity vector of $\Gamma(s, \tau)$. This velocity is the unknown that must be derived from the error criterion so that the solution $\Gamma(., \tau)$ converges towards a curve achieving a local minimum and thus, hopefully, towards the boundary of the object to be segmented, as $\tau \rightarrow \infty$.

Let us now define more precisely the region and boundary functionals. Let $\mathcal{U}$ be a class of domains (open, regular bounded sets, i.e. $C^{2}$ ) of $\mathbb{R}^{n}$, and $\Omega$ an element of $\mathcal{U}$ of boundary $\partial \Omega$, which we sometimes denote $\Gamma$. A boundary functional, $J_{b}$, may be expressed as a boundary integral of some scalar function $g$ of image features:

$$
J_{b}(\partial \Omega)=\int_{\partial \Omega} g(\mathbf{x}) d \mathbf{a}(\mathbf{x})
$$

where $\partial \Omega$ is the boundary of the region and $d \mathbf{a}$ its area element. The derivation of this boundary functional is classical $[3,18]$ and leads to the following velocity vector:

$$
\mathbf{v}_{b}=[g(\mathbf{x}) \kappa-\nabla g(\mathbf{x}) \cdot \mathbf{N}] \mathbf{N}
$$

where $\mathbf{N}$ is the inward unit normal vector of $\Gamma$ and $\kappa$ its mean curvature. The idea is to use a local parametrization of $\Gamma$ to reduce (1) to a standard problem in the calculus of variations.

A region functional, $J_{r}$, may be expressed as an integral, in a domain $\Omega$ of $\mathcal{U}$, of some function $f$ of some region features:

$$
J_{r}(\Omega)=\int_{\Omega} f(\mathbf{x}, \Omega) d \mathbf{x}
$$

In that case, the computation of the velocity vector is not as easy since the set of image regions does not have a structure of vector space. Moreover, the scalar function $f$ in (2) is generally region-dependent. This dependency on the region must be taken into account when searching for a local minimum of the functional.

In the literature, the classical approach is based upon the idea of transforming all functionals into boundary functionals thereby reducing (through a local parametrization of the 
boundary) the problem of minimization to a standard problem in the calculus of variations from which the computation of the Gâteaux derivatives follows. We propose here an approach based upon the use of shape derivation tools and we study the case of region-dependent features. We first recall principles of shape derivation and we then apply these tools when $f$ is a region-dependent function involving region histograms.

Note that we propose a comparison between calculus of variation and shape derivation tools for the computation of the velocity vector in [16]. Also note that we could have added a dependency of $g$ on $\partial \Omega$, see [19] for details.

\section{Computation of the derivative using shape derivation tools}

In this part, we propose to introduce shape derivation tools $[20,21]$ for the computation of the derivative.

Since the set of all image regions, namely $\mathcal{U}$, is not a vector space, it is difficult to compute the derivative of the criterion with respect to the domain $\Omega$. To circumvent this problem, shape derivation methods propose to apply a family of transformations $T_{\tau}$, indexed by a real parameter $\tau \geq 0$, to $\Omega, \Omega(\tau)=T_{\tau}(\Omega)$. The region functional becomes a function of $\tau, J(\tau) \stackrel{\text { def }}{=} J(\Omega(\tau))$. The error criterion $J(\tau)$ is then derived with respect to $\tau$ using shape derivation principles. Let us introduce the transformations and recall a theorem giving relation between derivatives that will be helpful for derivation of region functionals.

\subsection{Introduction of transformations}

As it has already been pointed out, the optimization of the region functional (2) is difficult since the set of regular domains (regular open bounded sets) $\mathcal{U}$ of $\mathbb{R}^{n}$ does not have the structure of a vector space. Variations of a domain must then be defined in some way. Let us consider a reference domain $\Omega \in \mathcal{U}$ and the set $\hat{\mathcal{A}}$ of applications $T: \Omega \rightarrow \mathbb{R}^{n}$, which are at least as regular as homeomorphisms (i.e. one to one with $T$ and $T^{-1}$ continuous). We define

$$
\hat{\mathcal{A}}=\left\{T \text { one to one, } T, T^{-1} \in W^{1, \infty}\left(\Omega, \mathbb{R}^{n}\right)\right\}
$$

where:

$$
\begin{aligned}
& W^{1, \infty}\left(\Omega, \mathbb{R}^{n}\right)=\left\{T: \Omega \rightarrow \mathbb{R}^{n}\right. \text { such that } \\
& \left.T \in L^{\infty}\left(\Omega, \mathbb{R}^{n}\right) \text { and } \partial_{i} T \in L^{\infty}\left(\Omega, \mathbb{R}^{n}\right), i=1, \cdots, n\right\}
\end{aligned}
$$

Given a shape function $F: \mathcal{U} \rightarrow \mathbf{R}^{+}$, for $T \in \hat{\mathcal{A}}$, let us define $\hat{F}(T)=F(T(\Omega))$. The key point is that $W^{1, \infty}\left(\Omega, \mathbb{R}^{n}\right)$ is a Banach space. This allows us to define the notion of derivative with respect to the domain $\Omega$ as follows:
Definition $1 F$ is Gâteaux differentiable with respect to $\Omega$ if and only if $\hat{F}$ is Gâteaux differentiable with respect to $T$.

In order to compute Gâteaux derivatives with respect to $T$ we introduce a family of deformation $(T(\tau))_{\tau \geq 0}$ such that $T(\tau) \in \hat{\mathcal{A}}$ for $\tau \geq 0, T(0)=I d$, and $T(.) \in$ $C^{1}\left([0, A] ; W^{1, \infty}\left(\Omega, \mathbb{R}^{n}\right), A>0\right.$.

For a point $\mathbf{x} \in \Omega$, we denote:

$$
\begin{array}{ccc}
\mathbf{x}(\tau)=T(\tau, \mathbf{x}) & \text { with } & T(0, \mathbf{x})=\mathbf{x} \\
\Omega(\tau)=T(\tau, \Omega) & \text { with } & T(0, \Omega)=\Omega
\end{array}
$$

Let us now define the velocity vector field $\mathbf{V}$ corresponding to $T(\tau)$ as

$$
\mathbf{V}(\tau, \mathbf{x})=\frac{\partial T}{\partial \tau}(\tau, \mathbf{x}) \quad \forall \mathbf{x} \in \Omega \forall \tau \geq 0
$$

\subsection{Computation of the derivative}

We now introduce two main definitions:

Definition 2 The Gâteaux derivative of $J_{r}(\Omega)=$ $\int_{\Omega} f(\mathbf{x}, \Omega) d \mathbf{x}$ in the direction of $\mathbf{V}$, noted $\left\langle J_{r}^{\prime}(\Omega), \mathbf{V}\right\rangle$, is equal to:

$$
<J_{r}^{\prime}(\Omega), \mathbf{V}>=\lim _{\tau \rightarrow 0} \frac{J_{r}(\Omega(\tau))-J_{r}(\Omega)}{\tau}
$$

Definition 3 The shape derivative of $f(\mathbf{x}, \Omega)$, noted $f_{s}(\mathbf{x}, \Omega, \boldsymbol{V})$, is equal to:

$$
f_{s}(\mathbf{x}, \Omega, \mathbf{V})=\lim _{\tau \rightarrow 0} \frac{f(\mathbf{x}, \Omega(\tau))-f(\mathbf{x}, \Omega)}{\tau}
$$

\subsubsection{Relation between the Gâteaux derivative and the shape derivative}

The following theorem gives a relation between the Gâteaux derivative and the shape derivative for the region functional (2).

Theorem 1 The Gâteaux derivative of the functional $J_{r}(\Omega)=\int_{\Omega} f(\mathbf{x}, \Omega) d \mathbf{x}$ in the direction of $\mathbf{V}$ is the following:

$$
\begin{aligned}
& <J_{r}^{\prime}(\Omega), \mathbf{V}>= \\
& \int_{\Omega} f_{s}(\mathbf{x}, \Omega, \mathbf{V}) d \mathbf{x}-\int_{\partial \Omega} f(\mathbf{x}, \Omega)(\mathbf{V}(\mathbf{x}) \cdot \mathbf{N}(\mathbf{x})) d \mathbf{a}(\mathbf{x})
\end{aligned}
$$

where $\boldsymbol{N}$ is the unit inward normal to $\partial \Omega$ and da its area element.

The proof can be found in $[20,21]$. Note that Theorem 1 provides a necessary condition for a domain $\hat{\Omega}$ to be an extremum of $J(\Omega)$ :

$$
\int_{\hat{\Omega}} f_{s}(\mathbf{x}, \hat{\Omega}, \mathbf{V}) d \mathbf{x}-\int_{\partial \hat{\Omega}} f(\mathbf{x}, \hat{\Omega})(\mathbf{V}(\mathbf{x}) \cdot \mathbf{N}(\mathbf{x})) d \mathbf{a}(\mathbf{x})=0 \forall \mathbf{V}
$$




\subsection{Computation of the evolution equation of an active contour}

We now make good use of the previous results to derive the velocity vector of the active contour.

\subsubsection{Region-independent features}

We first consider the simple case where the function $f$ does not depend on $\Omega$, i.e. $f=f(\mathbf{x})$. In that case, the shape derivative $f_{s}$ is equal to zero and the Gâteaux derivative of $J_{r}$ is simply (Theorem 1 ):

$$
<J_{r}^{\prime}(\Omega), \mathbf{V}>=-\int_{\partial \Omega} f(\mathbf{x})(\mathbf{V}(\mathbf{x}) \cdot \mathbf{N}(\mathbf{x})) d \mathbf{a}(\mathbf{x})
$$

This leads to the following evolution equation for regionindependent descriptors:

$$
\Gamma_{\tau}=f \mathbf{N} \quad \text { with } \quad \Gamma(\tau=0)=\Gamma_{0} .
$$

This is the classical result $[6,9]$ when $f$ has no region dependency. Let us now consider the more general case where the function $f$ has some region dependency.

\subsubsection{General case}

As far as the general case is considered, we have to express the domain integral of the shape derivative, i.e. $\int_{\Omega} f_{s}(\mathbf{x}, \hat{\Omega}, \mathbf{V}) d \mathbf{x}$, as a boundary integral which makes appear the normal velocity $(\mathbf{V} \cdot \mathbf{N})$. In order to do so, we can model the region feature $f$ as a linear combination of domain integrals as it has been done in [15], which is suitable for many region features such as functions of the mean or of the variance. In this case, using Theorem 1, the Gâteaux derivative in the direction of $\mathbf{V}$ of the functional $J_{r}$ defined in (2) is:

$$
\begin{aligned}
& <J_{r}^{\prime}(\Omega), \mathbf{V}>= \\
& \quad-\int_{\Gamma}(A(\mathbf{x}, \Omega)+f(\mathbf{x}, \Omega))(\mathbf{V}(\mathbf{x}) \cdot \mathbf{N}(\mathbf{x})) d \mathbf{a}(\mathbf{x})
\end{aligned}
$$

where $A(\mathbf{x}, \Omega)$ are some terms coming from the dependency on the region of $f$ whose expression is given in [15]. This leads to the following evolution equation for regiondependent descriptors:

$$
\Gamma_{\tau}=(A+f) \mathbf{N} \quad \text { with } \quad \Gamma(\tau=0)=\Gamma_{0} .
$$

In this article, we extend the computation of the evolution equation fo region-based functions $f$ depending on a continuous family of region criteria. We more particularly focus on the minimization of the distance between regions probability density functions.

\section{Matching histograms}

A natural way of generalizing the use of statistical image feature for image segmentation is to consider the full probability distribution of the feature of interest within the region, e.g. intensity, color, texture, etc... It turns out that in attempting to do so, one is naturally led to extend the criterion (2) to the case where the function $f$ depends on a continuous family of region criteria.

\subsection{Histograms estimation}

Consider a function $\mathbf{h}: \mathbb{R}^{n} \rightarrow \mathbb{R}^{m}$ which describes the feature of interest. Suppose we have learnt the probability density function (pdf) of the feature $\mathbf{h}$ within the image region of interest, and let $q(\boldsymbol{\alpha})$ be this pdf. Given a region $\Omega$, we can estimate the pdf of the feature $\mathbf{h}$ through the use of the Parzen method [22]: let $p: \mathbb{R}^{m} \rightarrow \mathbb{R}^{+}$be the Parzen window, a smooth positive function whose integral is equal to 1 . For the sake of simplicity but without loss of generality, we assume that $p$ is an $m$-dimensional Gaussian with 0 -mean and variance $\sigma^{2}$, we note

$$
p(\boldsymbol{\alpha})=g_{\sigma}(\boldsymbol{\alpha})=\frac{1}{\left(2 \pi \sigma^{2}\right)^{m / 2}} \exp \left(-\frac{|\boldsymbol{\alpha}|^{2}}{2 \sigma^{2}}\right),
$$

and we define

$$
\hat{q}(\boldsymbol{\alpha}, \Omega)=\frac{1}{K(\Omega)} \int_{\Omega} g_{\sigma}(\mathbf{h}(\mathbf{x})-\boldsymbol{\alpha}) d \mathbf{x},
$$

where $\mathbf{h}(\mathbf{x})$ is the value of the feature of interest at the point $\mathbf{x}$ of $\Omega$ and $K$ is a normalizing constant, in general depending of $\Omega$, such that $\int_{\mathbb{R}^{m}} \hat{q}(\boldsymbol{\alpha}, \Omega) d \boldsymbol{\alpha}=1$. Therefore

$$
K(\Omega)=\int_{\Omega} \int_{\mathbb{R}^{m}} g_{\sigma}(\mathbf{h}(\mathbf{x})-\boldsymbol{\alpha}) d \boldsymbol{\alpha} d \mathbf{x}=|\Omega|
$$

\subsection{Minimizing distance between histograms}

We next assume that we have a function $\varphi: \mathbb{R}^{+} \times \mathbb{R}^{+} \rightarrow$ $\mathbb{R}^{+}$which allows us to compare two pdfs. This function is small if the pdfs are similar and large otherwise. It allows us to introduce the following functional which represents the "distance" between the current histogram $\hat{q}(\boldsymbol{\alpha}, \Omega)$ and the reference one $q(\boldsymbol{\alpha})$ :

$$
D(\Omega)=\int_{\mathbb{R}^{m}} \varphi(\hat{q}(\boldsymbol{\alpha}, \Omega), q(\boldsymbol{\alpha})) d \boldsymbol{\alpha}
$$

The distance can be the Hellinger distance:

$$
\varphi(\hat{q}(\boldsymbol{\alpha}, \Omega), q(\boldsymbol{\alpha}))=(\sqrt{\hat{q}(\boldsymbol{\alpha}, \Omega)}-\sqrt{q(\boldsymbol{\alpha})})^{2},
$$

or the non symmetric chi-2 comparison function:

$$
\varphi(\hat{q}(\boldsymbol{\alpha}, \Omega), q(\boldsymbol{\alpha}))=\frac{(\hat{q}(\boldsymbol{\alpha}, \Omega)-q(\boldsymbol{\alpha}))^{2}}{q(\boldsymbol{\alpha})} .
$$


Using the tools developed in section 3, we compute the Gâteaux derivative of the functional $D$. We have the

Theorem 2 The Gâteaux derivative in the direction $\mathbf{V}$ of the functional $D$ defined in (3) is:

$$
\begin{aligned}
& <D^{\prime}(\Omega), \mathbf{V}>= \\
& -\frac{1}{|\Omega|} \int_{\Gamma}\left(\partial_{1} \varphi(\hat{q}(.), q(.)) * g_{\sigma}(\mathbf{h}(\mathbf{x}))-C(\Omega)\right)(\mathbf{V} \cdot \mathbf{N}) d \mathbf{a}(\mathbf{x})
\end{aligned}
$$

where $C(\Omega)=\int_{\mathbb{R}^{m}} \partial_{1} \varphi(\hat{q}(\boldsymbol{\alpha}, \Omega), q(\boldsymbol{\alpha})) \hat{q}(\boldsymbol{\alpha}, \Omega) d \boldsymbol{\alpha}$ and $\partial_{1} \varphi(.,$.$) the partial derivative of \varphi(r,$.$) according to the$ first variable $r$.

\section{Proof :}

By definition of $D$ we have

$$
<D^{\prime}(\Omega), \mathbf{V}>=\int_{\mathbb{R}^{m}}<(\varphi(\hat{q}(\boldsymbol{\alpha}, \Omega), q(\boldsymbol{\alpha})))^{\prime}, \mathbf{V}>d \boldsymbol{\alpha}
$$

Let us compute the Gâteaux derivative of $\varphi(\hat{q}(\boldsymbol{\alpha}, \Omega), q(\boldsymbol{\alpha}))$. We define:

$$
\varphi(\hat{q}(\boldsymbol{\alpha}, \Omega), q(\boldsymbol{\alpha}))=f\left(G_{1}(\boldsymbol{\alpha}, \Omega), G_{2}(\Omega)\right)
$$

where:

$$
\begin{aligned}
G_{1}(\boldsymbol{\alpha}, \Omega) & =\int_{\Omega} g_{\sigma}(\mathbf{h}(\mathbf{x})-\boldsymbol{\alpha}) d \mathbf{x} \\
H_{1}(\boldsymbol{\alpha}, \mathbf{x}) & =g_{\sigma}(\mathbf{h}(\mathbf{x})-\boldsymbol{\alpha}) \\
G_{2}(\Omega) & =|\Omega|=\int_{\Omega} d \mathbf{x}
\end{aligned}
$$

We obtain:

$$
\begin{gathered}
<f^{\prime}, \mathbf{V}>=f_{G_{1}}<G_{1}^{\prime}, \mathbf{V}>+f_{G_{2}}<G_{2}^{\prime}, \mathbf{V}>= \\
\frac{\partial_{1} \varphi(\hat{q}(\boldsymbol{\alpha}, \Omega), q(\boldsymbol{\alpha}))}{|\Omega|}\left(<G_{1}^{\prime}, \mathbf{V}>-\hat{q}(\boldsymbol{\alpha}, \Omega)<G_{2}^{\prime}, \mathbf{V}>\right)
\end{gathered}
$$

and, using Theorem 1:

$$
\begin{aligned}
& \quad<f^{\prime}, \mathbf{V}>= \\
& -\frac{\partial_{1} \varphi(\hat{q}(\boldsymbol{\alpha}, \Omega), q(\boldsymbol{\alpha}))}{|\Omega|} \int_{\Gamma}\left(H_{1}(\boldsymbol{\alpha}, \mathbf{x})-\hat{q}(\boldsymbol{\alpha}, \Omega)\right)(\mathbf{V} \cdot \mathbf{N}) d \mathbf{a}(\mathbf{x})
\end{aligned}
$$

Plugging this result into the expression of $\left\langle D^{\prime}(\Omega), \mathbf{V}\right\rangle$ and swapping the order of integration, we obtain

$$
\begin{aligned}
& <D^{\prime}(\Omega), \mathbf{V}>= \\
& -\frac{1}{|\Omega|} \int_{\Gamma}\left(\int_{\mathbb{R}^{m}} g_{\sigma}(\mathbf{h}(\mathbf{x})-\boldsymbol{\alpha}) \partial_{1} \varphi(\hat{q}(\boldsymbol{\alpha}, \Omega), q(\boldsymbol{\alpha})) d \boldsymbol{\alpha}\right. \\
& \left.-\int_{\mathbb{R}^{m}} \partial_{1} \varphi(\hat{q}(\boldsymbol{\alpha}, \Omega), q(\boldsymbol{\alpha})) \hat{q}(\boldsymbol{\alpha}, \Omega) d \boldsymbol{\alpha}\right)(\mathbf{V} \cdot \mathbf{N}) d \mathbf{a}(\mathbf{x})
\end{aligned}
$$

The first integral on the right-hand side is the convolution $\partial_{1} \varphi(\hat{q}(),. q()). * g_{\sigma}$ of the function $\partial_{1} \varphi(\hat{q}(),. q()):. \mathbb{R}^{m} \rightarrow$ $\mathbb{R}$ with the function $g_{\sigma}$. The final result is

$$
\begin{aligned}
& <D^{\prime}(\Omega), \mathbf{V}>= \\
& -\frac{1}{|\Omega|}\left[\int_{\Gamma}\left(\partial_{1} \varphi(\hat{q}(.), q(.)) * g_{\sigma}(\mathbf{h}(\mathbf{x}))\right)(\mathbf{V} \cdot \mathbf{N}) d \mathbf{a}(\mathbf{x})\right. \\
& \left.\quad-\int_{\Gamma} C(\Omega)(\mathbf{V} \cdot \mathbf{N}) d \mathbf{a}(\mathbf{x})\right]
\end{aligned}
$$

where $C(\Omega)=\int_{\mathbb{R}^{m}} \partial_{1} \varphi(\hat{q}(\boldsymbol{\alpha}, \Omega), q(\boldsymbol{\alpha})) \hat{q}(\boldsymbol{\alpha}, \Omega) d \boldsymbol{\alpha}$

\subsection{Generalization to second order histograms}

A further generalization of the previous case is to consider second order histograms which describe the probability of having the value $\boldsymbol{\alpha}_{1}$ at pixel $\mathbf{x}$ and the value $\boldsymbol{\alpha}_{2}$ at pixel $\mathbf{x}+\delta$, where $\delta$ is a fixed (usually small) vector of $\mathbf{R}^{n}$. This has been used very much in computer vision for analysing textures [23]. The corresponding pdf, noted $q_{\delta}\left(\boldsymbol{\alpha}_{1}, \boldsymbol{\alpha}_{2}\right)$ can be estimated with the same Parzen window technique. We define

$$
\begin{aligned}
& \hat{q}_{\boldsymbol{\delta}}\left(\boldsymbol{\alpha}_{1}, \boldsymbol{\alpha}_{2}, \Omega\right)= \\
& \quad \frac{1}{K_{\boldsymbol{\delta}}(\Omega)} \int_{\Omega} g_{\sigma}\left(\mathbf{h}(\mathbf{x})-\boldsymbol{\alpha}_{1}\right) g_{\sigma}\left(\mathbf{h}(\mathbf{x}+\boldsymbol{\delta})-\boldsymbol{\alpha}_{2}\right) d \mathbf{x}
\end{aligned}
$$

The normalizing constant $K_{\delta}(\Omega)$ is given by

$$
\begin{aligned}
& K_{\boldsymbol{\delta}}(\Omega)= \\
& \int_{\Omega} \int_{\mathbb{R}^{m} \times \mathbb{R}^{m}} g_{\sigma}\left(\mathbf{h}(\mathbf{x})-\boldsymbol{\alpha}_{1}\right) g_{\sigma}\left(\mathbf{h}(\mathbf{x}+\boldsymbol{\delta})-\boldsymbol{\alpha}_{2}\right) d \boldsymbol{\alpha}_{1} d \boldsymbol{\alpha}_{2} d \mathbf{x}
\end{aligned}
$$

And so, $K_{\delta}(\Omega)=|\Omega|$. We therefore define

$$
D_{\delta}(\Omega)=\int_{\mathbb{R}^{m} \times \mathbb{R}^{m}} \varphi\left(\hat{q}_{\delta}\left(\boldsymbol{\alpha}_{1}, \boldsymbol{\alpha}_{2}, \Omega\right), q_{\delta}\left(\boldsymbol{\alpha}_{1}, \boldsymbol{\alpha}_{2}\right)\right) d \boldsymbol{\alpha}_{1} d \boldsymbol{\alpha}_{2}
$$

For second-order histograms we have the

Theorem 3 The Gâteaux derivative in the direction $\mathbf{V}$ of the functional $D_{\delta}$ defined in (4) is:

$$
\begin{gathered}
<D_{\boldsymbol{\delta}}^{\prime}(\Omega), \mathbf{V}>=-\frac{1}{|\Omega|}\left[-\int_{\Gamma} C_{\boldsymbol{\delta}}(\Omega)\right)(\mathbf{V} \cdot \mathbf{N}) d \mathbf{a}(\mathbf{x})+ \\
\left.\int_{\Gamma}\left(\partial_{1} \varphi\left(\hat{q}_{\boldsymbol{\delta}}(), q_{\boldsymbol{\delta}}()\right) *\left(g_{\sigma} \otimes g_{\sigma}\right)(\mathbf{h}(\mathbf{x}), \mathbf{h}(\mathbf{x}+\boldsymbol{\delta}))\right)(\mathbf{V} \cdot \mathbf{N}) d \mathbf{a}(\mathbf{x})\right]
\end{gathered}
$$

where: $\quad C_{\boldsymbol{\delta}}(\Omega)=\int_{\mathbb{R}^{m} \times \mathbb{R}^{m}} \partial_{1} \varphi\left(\hat{q}_{\boldsymbol{\delta}}(), q_{\boldsymbol{\delta}}()\right) \hat{q}_{\boldsymbol{\delta}}() d \boldsymbol{\alpha}_{1} d \boldsymbol{\alpha}_{2}$

and $g_{\sigma} \otimes g_{\sigma}\left(\boldsymbol{\alpha}_{1}, \boldsymbol{\alpha}_{2}\right)=g_{\sigma}\left(\boldsymbol{\alpha}_{1}\right) g_{\sigma}\left(\boldsymbol{\alpha}_{2}\right)$.

Proof : The proof is identical to that of Theorem 2 . 


\section{Color histograms: segmentation of regions in video sequences}

This work has been motivated by $[24,25]$ where the tracking algorithms take benefit of statistical color ditributions. We propose here to use active contours in order to fit exactly the shape of the object to be segmented. We consider a video sequence where each frame is represented by the color function $\mathbf{h}: \mathbb{R}^{2} \rightarrow \mathbb{R}^{2}$. The color space used is $(H, V)$ where $H$ stands for the hue and $V$ for the value ${ }^{1}$. The goal is to segment a reference region, given in the previous image of the sequence, into the current one by minimizing the distance between the reference histogram $q$ of the region in the previous image and the estimated histogram $\hat{q}$ in the current frame. From an initial curve chosen by the user in the current frame, we want to make an active contour evolve towards the region in the current frame whose histogram is closest to the reference histogram of the previous frame.

In order to introduce a competition between the region of interest and the background region, we also consider the complement $\Omega^{c}$ of the region $\Omega$ of interest. They share the same boundary, $\Gamma$, but with normals pointing in opposite directions. We note $q^{c}$ the reference histogram of $\Omega^{c}$ and we look for the region $\Omega$ which minimizes the following criterion $^{2}$ :

$$
J(\Omega)=D(\Omega)+D\left(\Omega^{c}\right)+\lambda \int_{\Gamma} d s
$$

In this criterion, the first two terms are region functionals while the last one is a boundary functionals. The last one minimizes the curve length and is a regularization term weighted by the positive parameter $\lambda$. We have of course:

$$
\begin{array}{r}
D(\Omega)=\int_{\mathbb{R}^{2}} \varphi(\hat{q}(\boldsymbol{\alpha}, \Omega), q(\boldsymbol{\alpha})) d \boldsymbol{\alpha} \\
D\left(\Omega^{c}\right)=\int_{\mathbb{R}^{2}} \varphi\left(\hat{q}\left(\boldsymbol{\alpha}, \Omega^{c}\right), q^{c}(\boldsymbol{\alpha})\right) d \boldsymbol{\alpha}
\end{array}
$$

Computation of the Gâteaux derivative A straightforward application of Theorem 2 yields

$$
\begin{aligned}
& \quad<D^{\prime}(\Omega), \mathbf{V}>= \\
& -\frac{1}{|\Omega|} \int_{\Gamma}\left(\partial_{1} \varphi(\hat{q}(., \Omega), q(.)) * g_{\sigma}(\mathbf{h}(\mathbf{x}))-C(\Omega)\right)(\mathbf{V} \cdot \mathbf{N}) d s \\
& \text { with: } C(\Omega)=\int_{\mathbb{R}^{m}} \partial_{1} \varphi(\hat{q}(\boldsymbol{\alpha}, \Omega), q(\boldsymbol{\alpha})) \hat{q}(\boldsymbol{\alpha}, \Omega) d \boldsymbol{\alpha} .
\end{aligned}
$$

\footnotetext{
${ }^{1}$ We ignore the saturation to avoid the curse of dimensionality.

${ }^{2}$ The results are even better if we introduce the region area in the criterion by minimizing $D(\Omega)|\Omega|+D\left(\Omega^{c}\right)\left|\Omega^{c}\right|+\lambda s \int_{\Gamma} d$.
}

Similar results hold for $\Omega^{c}$ :

$$
\begin{aligned}
& <D^{\prime}\left(\Omega^{c}\right), \mathbf{V}>= \\
& \frac{1}{\left|\Omega^{c}\right|} \int_{\Gamma}\left(\partial_{1} \varphi\left(\hat{q}\left(., \Omega^{c}\right), q^{c}(.)\right) * g_{\sigma}(\mathbf{h}(\mathbf{x}))-C\left(\Omega^{c}\right)\right)(\mathbf{V} \cdot \mathbf{N}) d s \\
& C\left(\Omega^{c}\right)=\int_{\mathbb{R}^{m}} \partial_{1} \varphi\left(\hat{q}\left(\boldsymbol{\alpha}, \Omega^{c}\right), q^{c}(\boldsymbol{\alpha})\right) \hat{q}\left(\boldsymbol{\alpha}, \Omega^{c}\right) d \boldsymbol{\alpha}
\end{aligned}
$$

Computation of the evolution equation of an active contour It is well known that the minimization of the curve length leads to the Euclidean curve shortening flow $\lambda \kappa$ $[3,18]$. Then, from the previous derivatives, we can deduce the evolution of an active contour that will evolve towards a minimum of the criterion $J$ defined in (5). We find the following evolution equation:

$$
\Gamma_{\tau}=F \mathbf{N}
$$

$$
\begin{aligned}
& \text { with } \quad F=\frac{1}{|\Omega|}\left(\partial_{1} \varphi(\hat{q}(., \Omega), q(.)) * g_{\sigma}(\mathbf{h}(\mathbf{x}))-C(\Omega)\right) \\
& -\frac{1}{\left|\Omega^{c}\right|}\left(\partial_{1} \varphi\left(\hat{q}\left(., \Omega^{c}\right), q^{c}(.)\right) * g_{\sigma}(\mathbf{h}(\mathbf{x}))-C\left(\Omega^{c}\right)\right)+\lambda \kappa
\end{aligned}
$$

where $\kappa$ is the curvature of $\Gamma$ and $C(\Omega), C\left(\Omega^{c}\right)$ are given by equations (6) and (7), respectively.

Let us take the example of the Hellinger distance, where $\partial_{1} \varphi(r, \boldsymbol{\alpha})=(\sqrt{r}-\sqrt{q(\boldsymbol{\alpha})}) \sqrt{r}$, we find for the velocity vector:

$$
\begin{gathered}
F=\lambda \kappa+\frac{1}{|\Omega|}\left(\frac{(\sqrt{\hat{q}(., \Omega)}-\sqrt{q(.)})}{\sqrt{\hat{q}(., \Omega)}} * g_{\sigma}(\mathbf{h}(\mathbf{x}))-C(\Omega)\right) \\
-\frac{1}{\left|\Omega^{c}\right|}\left(\frac{\left(\sqrt{\hat{q}\left(., \Omega^{c}\right)}-\sqrt{q^{c}(.)}\right)}{\sqrt{\hat{q}\left(., \Omega^{c}\right)}} * g_{\sigma}(\mathbf{h}(\mathbf{x}))-C\left(\Omega^{c}\right)\right)
\end{gathered}
$$

We note that, for each region, two terms appear in the velocity, a local one that compares the two histograms for the intensity of the current point $\mathbf{h}(\mathbf{x})$ and a global one $C(\Omega)$.

\subsection{Implementation}

As far as the numerical implementation is concerned, we use the level set method approach first proposed by Osher and Sethian [26] and applied to active contours in [27]. The key idea of the level set method is to introduce an auxiliary function $U(\mathbf{x}, \tau)$ such that $\Gamma(\tau)$ is the zero level set of $U$. The function $U$ is often chosen to be the signed distance function of $\Gamma(\tau)$. The evolution equation (8) then becomes:

$$
\frac{\partial U(\tau)}{\partial \tau}=F|\nabla U|
$$


The velocity function $F$ is computed only on the curve $\Gamma(\tau)$ but we can extend its expression to the whole image domain $\Omega$. To implement the level set method, solutions must be found to circumvent problems coming from the fact that the signed distance function $U$ is not a solution of the PDE (9); see [28] for details. In our work, the function $U$ is reinitialized so that it remains a distance function.

\subsection{Experimental results}

Experimental results have been obtained on the sequence "Erik" from the European group COST211.

Experiments are conducted using the chi-2 comparison fucntion with $\varphi(r, \alpha)=(r-q(\boldsymbol{\alpha}))^{2} / q(\boldsymbol{\alpha})$ and $\partial_{1} \varphi(r, \boldsymbol{\alpha})=$ $2(r-q)(\mathbf{q}) d)$

The region of interest is the face. We assume that it has been segmented in the first image as shown in Fig.1.a. The first two reference histograms are computed. These two histograms are represented in Fig.1.b using different colors for each of the two regions. The reference histogram for the face, $q$, is represented in red using an intensity depending on the value of the probability density function, while the reference histogram for the background, $q^{c}$, is shown in green. The two reference histograms are also given Fig.1.c for the background reference histogram $q^{c}$ and Fig.1.d for the object reference histogram, $q$. For a given region $\Omega$, and for a point $\boldsymbol{\alpha}=\left[\alpha_{1}, \alpha_{2}\right]^{T}$, the function $\hat{q}(\boldsymbol{\alpha}, \Omega)$ represents the probability to obtain $H(\mathbf{x})=\epsilon_{1} \quad 1$ and $V(\mathbf{x}) \notin \quad{ }_{2}$ for $\mathbf{x}$ belonging to the region $\Omega$.

Then, using the two reference histograms of the previous frame, we make the active contour evolve using equation (8) in the current frame. The initial curve is chosen to be a circle. The evolution of the active contour in the current frame is shown in Fig.2. We can notice that the final contour in Fig.2.c nicely describes the region of interest, and the face is accurately segmented. We can also visualize the evolution of the object histogram, $\hat{q}(\boldsymbol{\alpha}, \Omega)$, during the propagation of the active contour in Fig.2. The final object histogram given Fig.2.d can be compared to the reference object histogram Fig.1.d, showing an efficient minimization of the distance between the two histograms.

\section{Conclusion}

In this article, we have concentrated on the problem of finding local minima of a large class of region functionals by applying methods of shape derivation [20,21].

We have turned our attention to a new class of regionbased functionals by considering histograms of image features. The shape derivation tools have allowed us to easily derive the velocity field that defines the evolution of the region boundary.

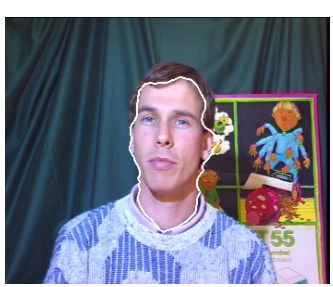

(a) Reference segmentation

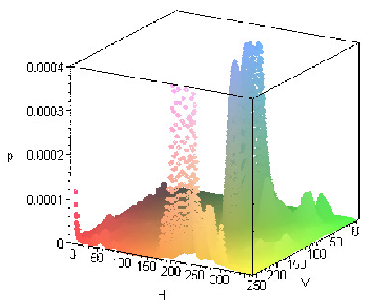

(c) Background reference

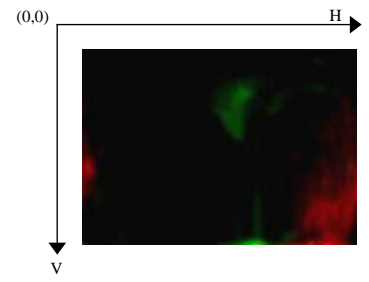

(b) Reference histograms

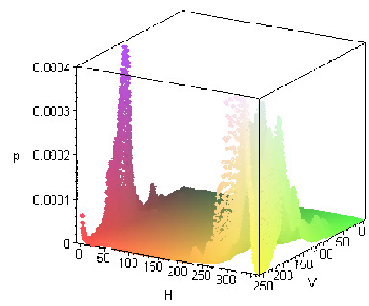

(d) Object reference
Figure 1. The reference segmentation of the previous frame (a), the two reference histograms, $q$, in red for the face, and $q^{c}$ in green for the background (b), the corresponding background reference histogram $q^{c}$ (c) and the corresponding object reference histogram $q(\mathrm{~d})$.

The final part of the paper has been devoted to an application of the previous methods to the problem of region segmentation with a given color histogram in a sequence of images. Our experimental results show that the technique has indeed some interesting potentials.

\section{References}

[1] Kass, M., Witkin, A., Terzopoulos, D.: Snakes: Active contour models. International Journal of Computer Vision 1 (1988) 321-332

[2] Cohen, L.: On active contour models and balloons. Computer Vision, Graphics and Image Processing : Image Understanding 53 (1991) 211-218

[3] Caselles, V., Kimmel, R., Sapiro, G.: Geodesic active contours. International Journal of Computer Vision 22 (1997) $61-79$

[4] Cohen, L., Bardinet, E., Ayache, N.: Surface reconstruction using active contour models. In: SPIE Conference on Geometric Methods in Computer Vision, San Diego, CA (1993)

[5] Ronfard, R.: Region-based strategies for active contour models. International Journal of Computer Vision 13 (1994) 229 251

[6] Zhu, S., Yuille, A.: Region competition: unifying snakes, region growing, and bayes/MDL for multiband image segmentation. IEEE Transactions on Pattern Analysis and Machine Intelligence 18 (1996) 884-900 


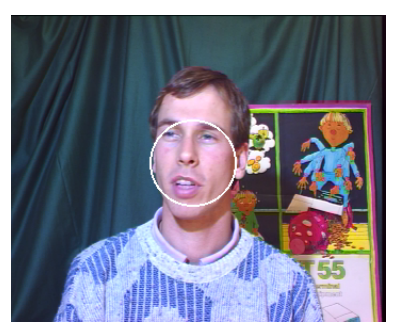

(a) Initial Contour
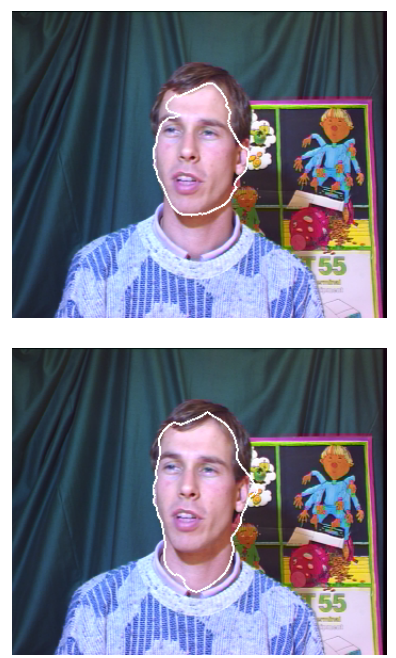

(c) Final contour

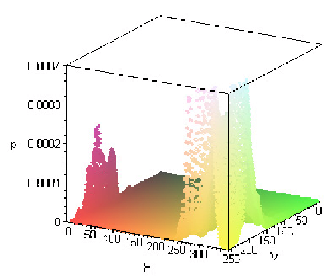

(b) Initial histogram $\hat{q}(\boldsymbol{\alpha}, \Omega)$
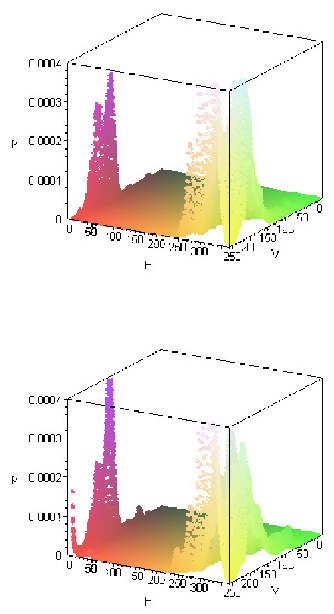

(d) Final histogram $\hat{q}(\boldsymbol{\alpha}, \Omega)$

Figure 2. Evolution of the region histogram $\hat{q}(\boldsymbol{\alpha}, \Omega)$ of the current frame during the evolution of the active contour

[7] Chakraborty, A., Staib, L., Duncan, J.: Deformable boundary finding in medical images by integrating gradient and region information. IEEE Transactions on Medical Imaging 15 (1996) 859-870

[8] Chesnaud, C., Réfrégier, P., Boulet, V.: Statistical region snake-based segmentation adapted to different physical noise models. IEEE Transactions on Pattern Analysis and Machine Intelligence 21 (1999) 1145-1156

[9] Paragios, N., Deriche, R.: Geodesic active regions: A new paradigm to deal with frame partition problems in computer vision. Journal of Visual Communication and Image Representation 13 (2002) 249-268

[10] Paragios, N., Deriche, R.: Geodesic active regions and level set methods for supervised texture segmentation. The International Journal of Computer Vision 46 (2002) 223

[11] Debreuve, E., Barlaud, M., Aubert, G., Darcourt, J.: Space time segmentation using level set active contours applied to myocardial gated SPECT. IEEE Transactions on Medical Imaging 20 (2001) 643-659

[12] Yezzi, A., Tsai, A., Willsky, A.: A statistical approach to snakes for bimodal and trimodal imagery. In: International Conference on Image Processing, Kobe Japan (1999)
[13] Chan, T., Vese, L.: Active contours without edges. IEEE Transactions on Image Processing 10 (2001) 266-277

[14] Jehan-Besson, S., Barlaud, M., Aubert, G.: Video object segmentation using eulerian region-based active contours. In: International Conference on Computer Vision, Vancouver, Canada (2001)

[15] Jehan-Besson, S., Barlaud, M., Aubert, G.: DREAM ${ }^{2}$ S: Deformable regions driven by an eulerian accurate minimization method for image and video segmentation. International Journal of Computer Vision 53 (2003) 45-70

[16] Aubert, G., Barlaud, M., Faugeras, O., Jehan-Besson, S.: Image segmentation using active contours: Calculus of variations or shape gradients ? SIAM Applied Mathematics (2003) to appear

[17] Kim, J., Fisher III, J., Yezzi, A., Cetin, M., Willsky, A.: Nonparametric methods for image segmentation using information theory and curve evolution. In: International Conference on Image Processing, Rochester, NY (2002)

[18] Kischenassamy, S., Kumar, A., Olver, P., Tannenbaum, A., Yezzi, A.: Conformal curvature flows: from phase transitions to active vision. Archive for Rational Mechanics and Analysis 134 (1996) 275-301

[19] Gastaud, M., Barlaud, M., Aubert, G.: Tracking video objects using active contours. In: WMVC, Orlando, FL (2002) 90-95

[20] Sokolowski, J., Zolésio, J.P.: Introduction to shape optimization. Shape sensitivity analysis. Volume 16 of Springer Ser. Comput. Math. Springer-Verlag, Berlin (1992)

[21] Delfour, M., Zolésio, J.P.: Shapes and geometries. Advances in Design and Control. Siam (2001)

[22] Duda, R., Hart, P.: Pattern Classification and Scene Analysis. John Wiley and Sons, Inc. (1973)

[23] Haralick, R., Shapiro, L.: Computer and Robot Vision. Volume 1 \& 2. Addison-Wesley (1992-1993)

[24] Comaniciu, D., Ramesh, V., Meer, P.: Real-time tracking of non-rigid objects using mean shift. In: Computer Vision and Pattern Recognition. Volume 2., Hilton Head, SC (2000) 142-149

[25] Chen, H., Liu, T.: Trust-region methods for real-time tracking. In: International Conference on Computer Vision. Volume 2., Vancouver, Canada (2001) 717-722

[26] Osher, S., Sethian, J.: Fronts propagating with curvaturedependent speed: Algorithms based on hamilton-jacobi formulation. Journal of Computational Physics 79 (1988) 12-49

[27] Caselles, V., Catte, F., Coll, T., Dibos, F.: A geometric model for active contours. Numerische Mathematik 66 (1993) 1-31

[28] Gomes, J., Faugeras, O.: Reconciling distance functions and level sets. Journal of Visual Communication and Image Representation 11 (2000) 209-223 